\title{
IDENTIFICACIÓN DE LOS SITIOS DE UNIÓN DEL PLOMO EN LA SUPERFICIE DEL ADSORBENTE DE RESIDUOS DE PELOS DE LA INDUSTRIA CURTIEMBRE
}

\author{
Carmen Carola Palomino Amorín ${ }^{* a}$, Lena Tellez Monzon, Lizardo Visitación Figueroa ${ }^{a}$
}

\section{RESUMEN}

La presente investigación tuvo como objetivo identificar los grupos funcionales que son responsables de la adsorción de $\mathrm{Pb}$ (II) sobre los residuos de pelos tratados del proceso de pelambre enzimático de las curtiembres. Para este propósito se realizó la preparación del adsorbente, que consistió en lavar los residuos de pelos con el fin de desencalar y bajar el pH de 12 a neutro, tras lo cual se secaron y se les aplicó el tratamiento de hidrólisis ácida, al término del cual estos fueron lavados, secados y molidos. Luego se colocó en agitación los pelos tratados con la solución de $\mathrm{Pb}(\mathrm{II})$ en un rango de tiempo y $\mathrm{pH}$, para el estudio de isotermas de adsorción y el estudio cinético. Al termino de los cuales se realizó una caracterización química, a través del análisis FTIR y del análisis DRX. Así también una caracterización física con un análisis SEM, por medio de dos detectores del equipo. Estos análisis se realizaron en tres muestras, siendo estos los residuos de pelos sin y con tratamiento y los residuos de pelos tratados usados en la adsorción de $\mathrm{Pb}$ (II). Los resultados obtenidos mostraron que los grupos funcionales responsables de la adsorción de $\mathrm{Pb}(\mathrm{II})$ son el carboxilo, amino y los grupos que contienen azufre $(\mathrm{S}=\mathrm{O})$, que se encuentran en la superficie del bioadsorbente.

Palabras clave: plomo, queratina, FTIR, bioadsorbente, bioadsorción, pelambre.

\section{IDENTIFICATION OF LEAD UNION SITES ON THE HAIR ADSORBENT SURFACE OF THE TANNERY INDUSTRY}

\begin{abstract}
The objective of this research was to identify the functional groups that are responsible for the adsorption of $\mathrm{Pb}$ (II) on the treated hair residues of the enzymatic unhairing process of the tanneries. For this purpose was made the preparation of adsorbent, which consisted in washing hair residues in order to deliming and lower the $\mathrm{pH}$ of 12 to neutral, after which they were dried and was applied these the treatment of acid hydrolysis, at the end of which these were washed, dried and ground. Then the hairs treated with the $\mathrm{Pb}$ (II) solution were placed in agitation in a range of time and $\mathrm{pH}$, for the study of adsorption isotherms and the kinetic

$\mathrm{a}^{*}$ Centro de Investigación en Química, Toxicología y Biotecnología Ambiental del Departamento de Química de la Facultad de Ciencias de la Universidad Nacional Agraria La Molina. Lima-Perú.

e-mail: carolapa16@gmail.com
\end{abstract}


study. At the end of which a chemical characterization was carried out through the FTIR analysis and the XRD analysis. Thus also a physical characterization with a SEM analysis, by means of two detectors of the equipment. These analyses were carried out in 3 samples being these residues of hairs without and with treatment and the residues of treated hairs used in the adsorption of $\mathrm{Pb}$ (II). The results obtained showed that the functional groups responsible for the adsorption of $\mathrm{Pb}$ (II) are the carboxyl, amino and the groups containing sulphur $(\mathrm{S}=\mathrm{O})$, which are on the surface of the bioadsorbent

Key word: lead, keratin, unhairing, FTIR, bioadsorbent, bioadsorption.

\section{INTRODUCCIÓN}

La presencia de plomo en el agua potable, incluso a bajas concentraciones, puede causar enfermedades tales como anemia, hepatitis y síndrome nefrótico ${ }^{1}$. El plomo es no biodegradable y puede acumularse en los tejidos vivos, con lo que se concentra en toda la cadena alimentaria y puede ser fácilmente absorbido por el cuerpo humano ${ }^{2}$. La contaminación del agua por plomo se debe a los efluentes industriales no tratados, generados por las fábricas de tetraetilo de plomo, pinturas, baterías, municiones, cerámicas de vidrio, minería, entre otros. El plomo es un metal pesado tóxico y, debido al daño que puede producir su ingesta, la Agencia de Protección Ambiental (EPA) lo ha clasificado entre las 20 sustancias químicas más peligrosas para la salud humana; asimismo, estableció el límite máximo permisible de plomo en agua potable de $0,001 \mathrm{ppm}^{3}$.

Existen distintas alternativas para remover el plomo en las aguas residuales como el ajuste del pH básico, coagulación sedimentación, ósmosis inversa, intercambio iónico y remoción con adsorbentes ${ }^{4}$. La adsorción, en comparación con otro método, parece ser un proceso atractivo a la vista de su eficiencia y la facilidad con la que se puede aplicar en el tratamiento de aguas residuales que contienen metales pesados 5 . Pero tiene una desventaja, el proceso de adsorción es caro debido a los altos costos de los adsorbentes y al proceso de regeneración. El uso de adsorbentes, a base de materiales orgánicos, surge como una alternativa industrialmente atractiva para remover metales; debido a sus ventajas en la capacidad de disminuir la concentración de iones metálicos a niveles de partes por billón (ppb), producida por su alta afinidad por los cationes metálicos principalmente, su fácil disponibilidad, así como por su relativo bajo costo de elaboración ${ }^{6}$.

Pelos y plumas son subproductos residuales de industrias, tales como las de curtido de pieles, las chacineras, los mataderos de pollos, etc. En la mayoría de los casos, estas biomasas, constituidas casi al $100 \%$ por la proteína más abundante en el planeta, es decir, la queratina, son incineradas o biodegradadas en vertederos sin llegar a ser utilizadas en diferentes procesos tecnológicos aplicados ${ }^{7}$.

Las proteínas de queratina son materiales potencialmente útiles para usar como adsorbentes o sistemas de filtración para la eliminación de contaminantes tóxicos, debido al número 
de grupos funcionales presentes en las cadenas principales y secundarias de las proteínas ${ }^{8}$, entre ellos tenemos a los grupos carboxilo (R-COOH), hidroxilo (R-OH), amino (R-NH2) y sulfhidrilo (R-SH). Estos grupos funcionales permiten la fuerte atracción de los iones metálicos a las materias queratínicas, dándoles propiedades bioadsorbentes ideales para eliminación de metales pesados de las aguas residuales ${ }^{9}$.

La presente investigación tiene como objetivo la identificación de los grupos funcionales que son responsables de la adsorción de $\mathrm{Pb}(\mathrm{II})$ sobre los residuos de pelos tratados del proceso de pelambre enzimático de la curtiembre.

\section{MATERIALES Y MÉTODOS}

Preparación del adsorbente: $2 \mathrm{~kg}$ de residuos de pelos del proceso de pelambre enzimático de la curtiembre fueron lavados con $2 \mathrm{~L}$ de agua a $28^{\circ} \mathrm{C}, 0,1 \mathrm{~kg}$ de sulfato de amonio y $0,01 \mathrm{~kg}$ de Tandesta ${ }^{\circledR}$ BASE (desencalante que contiene sales de hidrólisis ácida y ácidos complejantes de calcio) por un tiempo de 10 minutos con el fin de desencalar y bajar el pH 12 hasta neutro, luego se les agregó $0,02 \mathrm{~kg}$ de Tamplex ${ }^{\circledR}$ FLA (complejante de calcio) cada15 minutos para terminar de bajar el $\mathrm{pH}$ a 7 . Después se lavaron con agua, se escurrieron y finalmente se secaron a temperatura ambiente. A continuación, se aplicó el tratamiento de hidrólisis ácida a las muestras de pelos, el cual se realizó por incubación de estos con $\mathrm{HCl}$ $8 \mathrm{M}$ (15.1 cm3/g pelo) durante $16.5 \mathrm{~h}^{7}$. Tras el tratamiento de hidrólisis, los pelos se lavaron repetidas veces con agua destilada y luego se secaron a temperatura ambiente. Por último, los pelos se pasaron por el cernidor, con el fin de que no se presenten grumos en la muestra.

Caracterización del adsorbente: Los residuos de pelos, provenientes del pelambre enzimático de la curtiembre, fueron analizados antes y después del tratamiento de hidrólisis ácida; asimismo, los pelos tratados también fueron analizados después de la adsorción de $\mathrm{Pb}^{2+}$, para los cuales se utilizó un espectrofotómetro infrarrojo con transformada de Fourier (FTIR) SHIMADZU IRPrestige-21, con un espectro de medición entre 4500 a $500 \mathrm{~cm}^{-1}$ y con una resolución de $4 \mathrm{~cm}^{-1}$ determinándose los grupos funcionales presentes en el adsorbente. También se realizó la caracterización por medio de la técnica de difracción de rayos X (DRX) usando un difractómetro de rayos X (SHIMADZU XRD-7000) con $40 \mathrm{Kv}, 30 \mathrm{~mA}$ y un ánodo de cobre $(\mathrm{Cu})$. Los datos fueron tomados en un rango de barrido $(2 \theta)$ de $3^{\circ}$ hasta $70^{\circ}$. Por otro lado, la caracterización morfológica se determinó usando el detector de electrones secundarios del microscopio electrónico de barrido (SEM) JEOL (JSM-6390LV). Adicionalmente, por el detector de electrones retrodispersados del mismo equipo, se determinó la imagen de composición, que es una micrografía que proporciona una idea acerca de la composición de la muestra basándose en el contraste de las distintas zonas que conforman la superficie del material.

Proceso de bioadsorción: Se realizó pruebas de adsorción tipo batch, en los cuales se realizó el estudio de las isotermas de adsorción, para lo cual se colocaron en vasos de precipitación $50 \mathrm{ml}$ de soluciones de $\mathrm{Pb}^{2+}$ de 25, 50, 125, 250, 500, 700 y 1000 ppm. A cada solución se 
agregó $0,1 \mathrm{~g}$ de residuos de pelos tratados del pelambre enzimático, se ajustaron a pH óptimo $(\mathrm{pH}=7)$ y se mantuvieron en agitación durante 30 minutos $^{10}$. Así también, se realizó el estudio cinético, para lo cual se colocaron $15 \mathrm{ml}$ de una solución de $3000 \mathrm{ppm}^{2+}$ en vasos de precipitación y se les agregó $0,1 \mathrm{~g}$ de residuos de pelos tratados de pelambre enzimático, a los cuales se les reguló a un $\mathrm{pH}$ óptimo $(\mathrm{pH}=7)$ y se mantuvo las mezclas en agitación por periodos determinados $(0,5,10,15,30,60,90,120,240,360 \text { y } 480 \mathrm{~min})^{6}$. Luego, para cada estudio, las soluciones fueron filtradas y la solución resultante fue analizada para determinar la concentración del $\mathrm{Pb}^{2+}$.

\section{RESULTADOS Y DISCUSIÓN}

\section{Caracterización física del adsorbente}

\section{Análisis por microscopía electrónica de barrido (SEM)}

La caracterización morfológica se realizó con el SEM a través del detector de electrones secundarios, por lo que en la figura 1 se muestra las micrografías obtenidas de los residuos de pelos tratados (hidrólisis ácida) del pelambre enzimático y de estos después de la adsorción del $\mathrm{Pb}(\mathrm{II})$. Como se puede observar, la morfología de la superficie de los residuos de pelos tratados (figura 1A) cambió significativamente después de la adsorción de $\mathrm{Pb}$ (II) (figuras $1 \mathrm{~B}$ y 1C) mostrando una superficie cubierta de una capa rugosa o granular con respecto a antes de la adsorción. Lo que indica que la formación de esta superficie se debe a la unión del Pb(II) con la superficie del bioadsorbente.
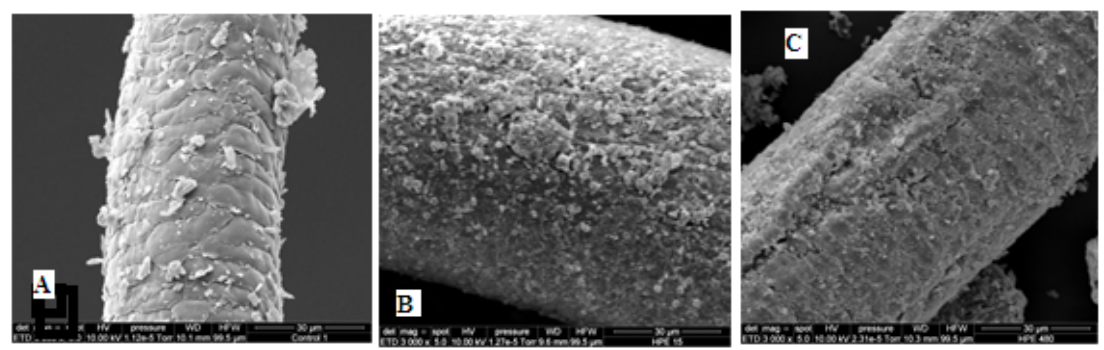

Figura 1. Micrografías obtenidas del SEM con el detector de electrones secundarios: (A) Residuos de pelos tratados del pelambre enzimático antes de la adsorción de $\mathrm{Pb}(\mathrm{II})$; (B) y (C) Residuos de pelos tratados después de la adsorción de $\mathrm{Pb}(\mathrm{II})$. Aumento 3000x.

Lo anterior es confirmado con la información dada con el detector de electrones retrodispersados del SEM, el cual permite establecer dos zonas en la superficie de una muestra; una zona obscura, que significa la presencia de elementos con un bajo número atómico (elementos ligeros) y una zona más brillante, que representa elementos de alto número atómico (elementos pesados). Esto se observó en las figuras $2 \mathrm{~B}^{*}$ y $2 \mathrm{C}^{*}$, en las que se muestra que la superficie de los residuos de pelos tratados después de la adsorción de $\mathrm{Pb}$ (II) 
presentó zonas brillantes, lo que indicaría la presencia de elementos con número atómico alto, como el caso del plomo (metal pesado). Esto no se observó en la superficie de los pelos tratados antes de la adsorción de $\mathrm{Pb}(\mathrm{II})$, la cual se presentó de manera homogénea, es decir, no había zonas brillantes (figura 2A*).
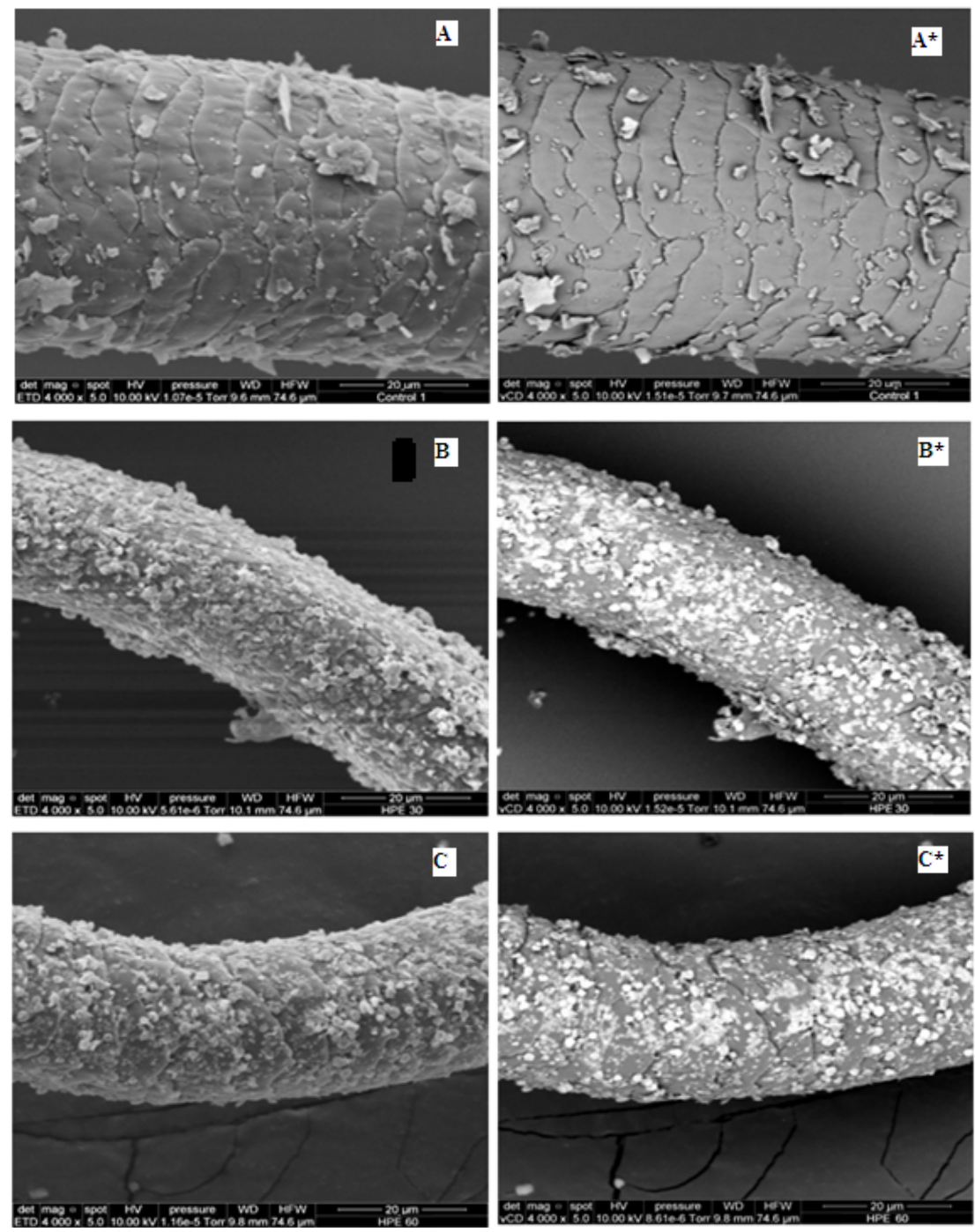

Figura 2. Micrografías obtenidas por el microscopio electrónico de barrido con el detector de electrones secundarios (columna izquierda) y con el detector de electrones retrodispersados (columna derecha): (A) y ( $\left.\mathrm{A}^{*}\right)$ corresponden a los residuos de pelos tratados del pelambre enzimático antes de ser utilizados en la adsorción de $\mathrm{Pb}(\mathrm{II})$; (B), y (B*), (C) y ( $\left.\mathrm{C}^{*}\right)$ corresponden a los residuos de pelos tratados después de la adsorción de $\mathrm{Pb}(\mathrm{II})$. Se realizaron con un aumento de $4000 \mathrm{x}$ 


\section{Caracterización química del adsorbente}

\section{Análisis de espectroscopía infrarroja por transformada de Fourier (FTIR)}

Los resultados del estudio de los espectros infrarrojos de los residuos de pelos del pelambre enzimático de la curtiembre antes y después del tratamiento de hidrólisis ácida y del espectro infrarrojo de los residuos de pelos con tratamiento después del proceso de adsorción de $\mathrm{Pb}(\mathrm{II})$ se muestran en las figuras 3,4 y 5 , respectivamente, y en el cuadro 1 .

En las figuras 3, 4 y 5 se observan las bandas del espectro infrarrojo, características de la queratina en los picos a 1631,78 y 1633,21 cm-1 (Amida I), 1519.91, 1516,05 y 1515,64 $\mathrm{cm}^{-1}$ (Amida II) y 1238,30 y $1237,38 \mathrm{~cm}^{-1}$ (Amida III).

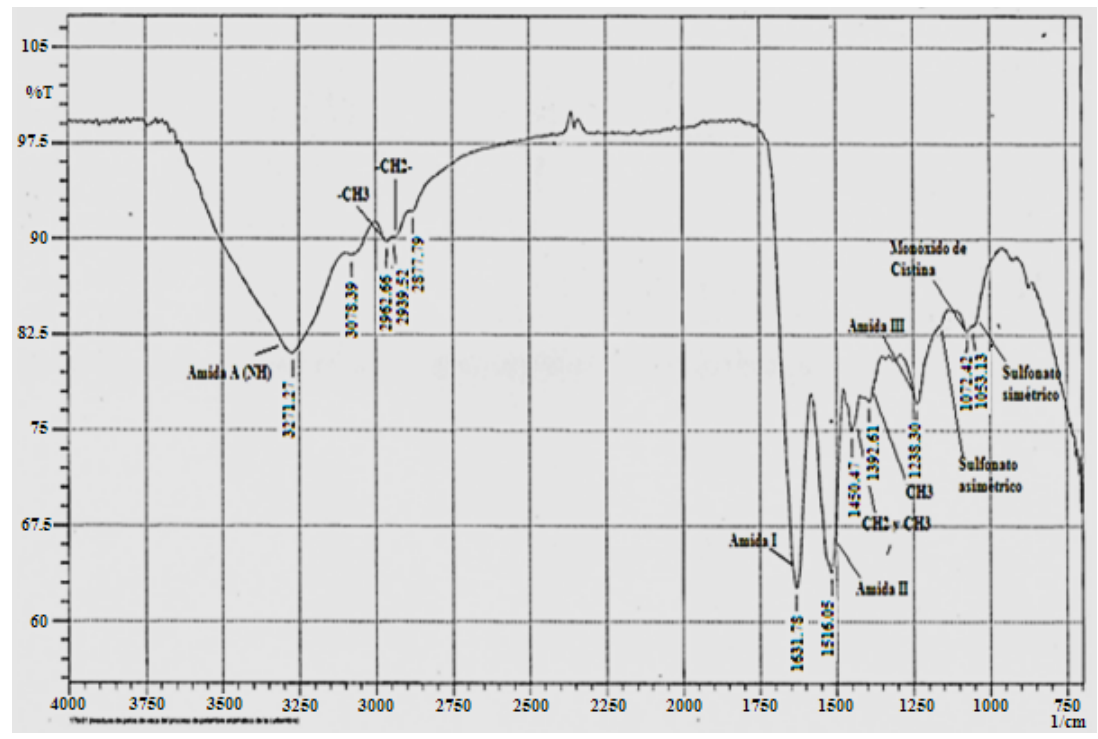

Figura 3. Espectro infrarrojo de los residuos de pelos del proceso de pelambre enzimático de la curtiembre. 


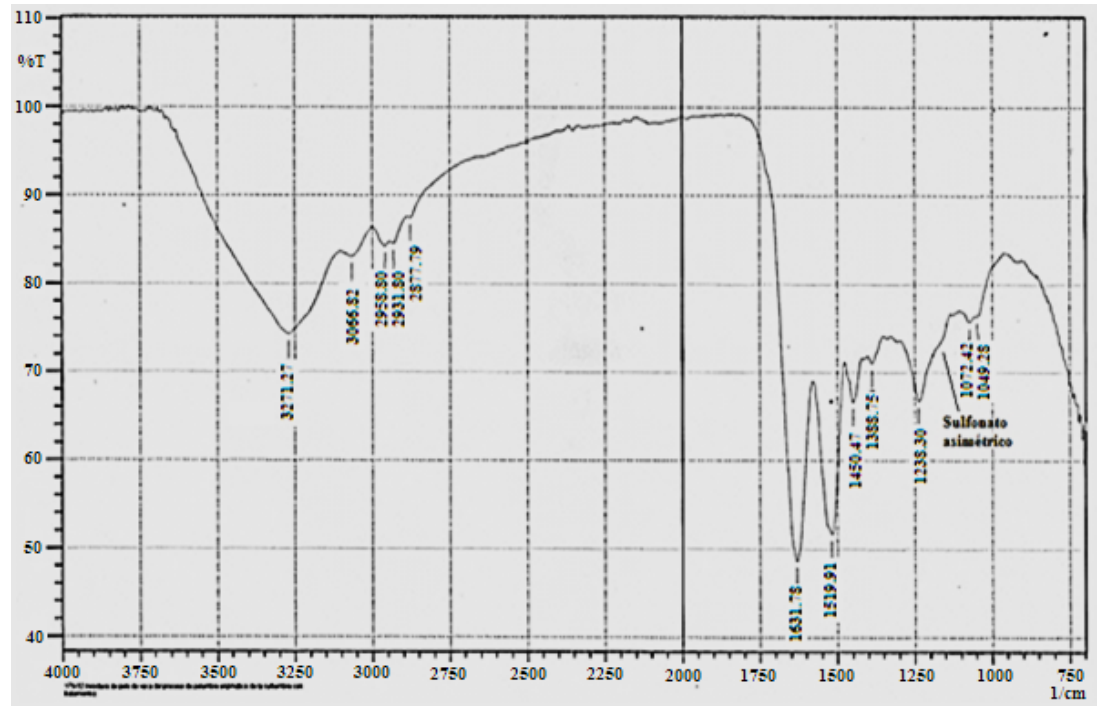

Figura 4. Espectro infrarrojo de los residuos de pelos tratados del proceso de pelambre enzimático de la curtiembre.

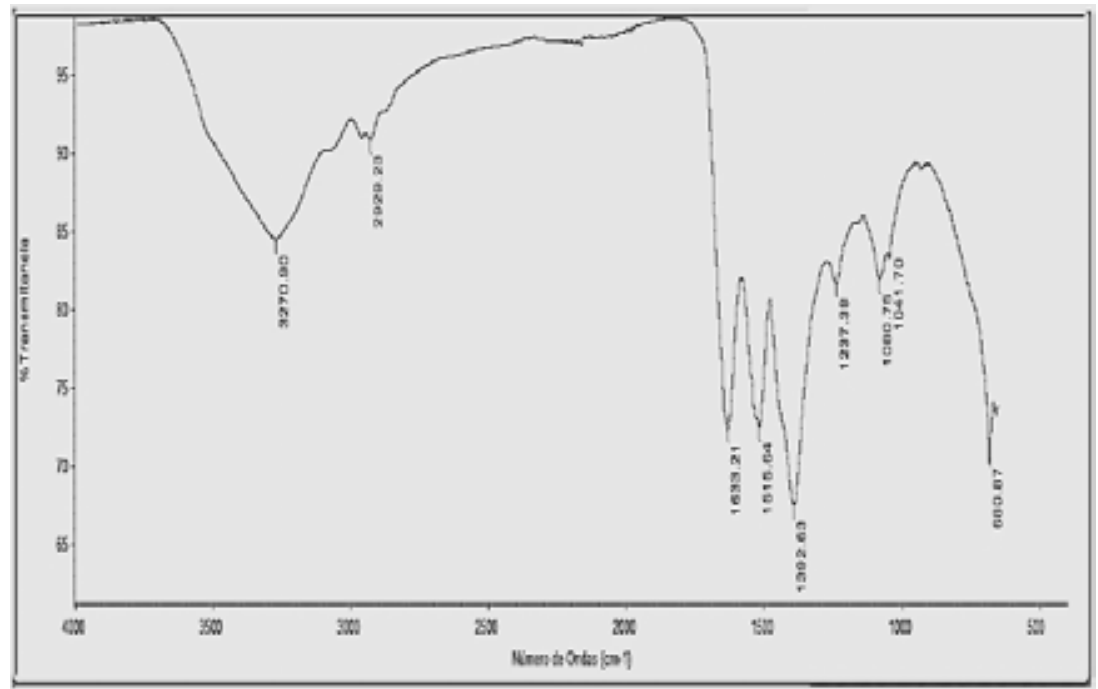

Figura 5. Espectro infrarrojo de los residuos de pelos tratados del proceso de pelambre enzimático de la curtiembre después de la adsorción del $\mathrm{Pb}(\mathrm{II})$. 
En el cuadro 1 se recogen los picos encontrados en los tres espectros infrarrojos, junto con su posición (número de onda) en cm-1 y los posibles grupos funcionales asignados para cada pico.

Cuadro 1. Bandas de los espectros infrarrojos de los residuos de pelos del proceso de pelambre enzimático y asignaciones.

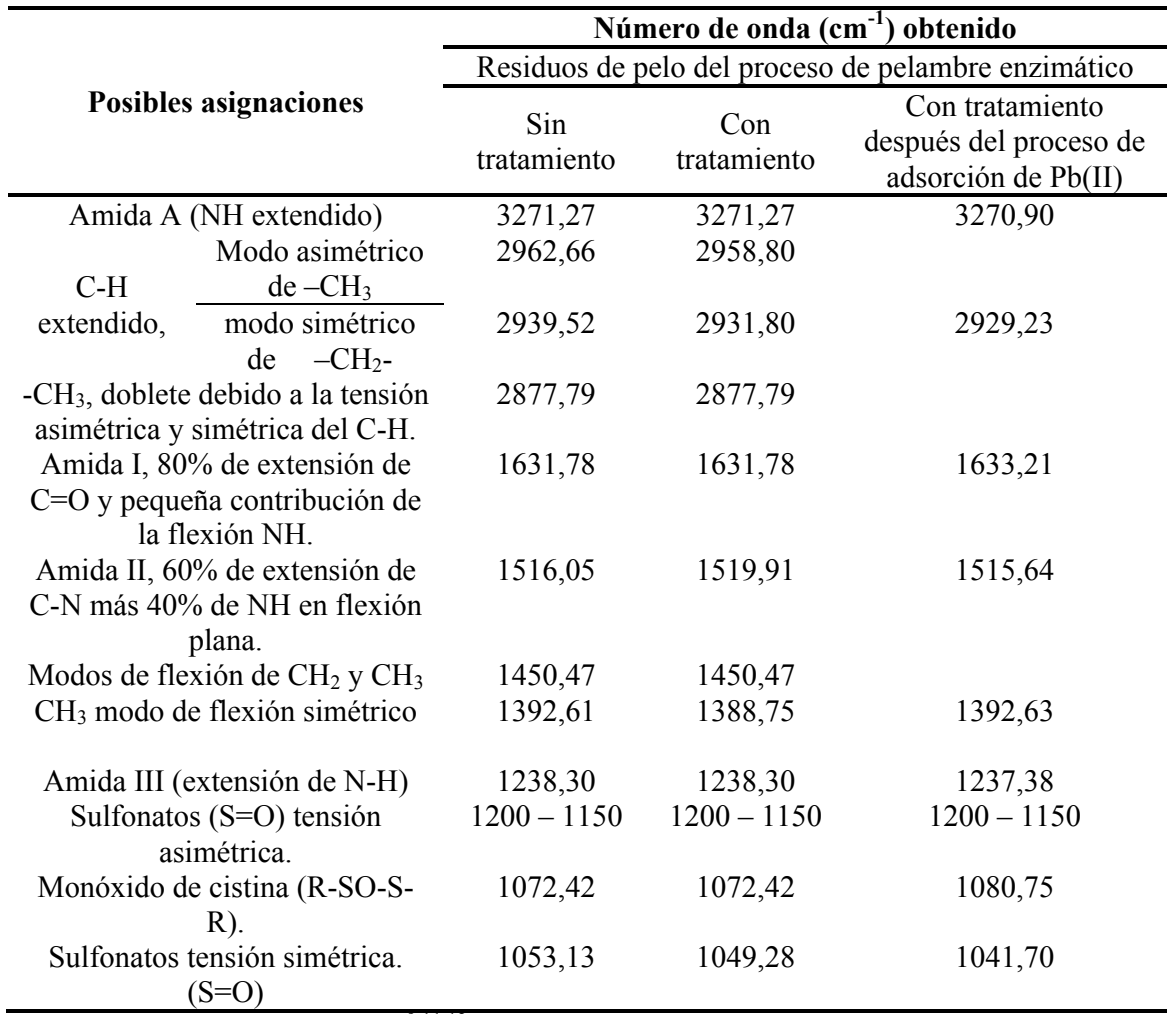

Fuente: Elaborado con base en ${ }^{9,11,12}$

A partir del análisis FTIR, de los residuos de pelos del pelambre enzimático antes y después de ser tratados y de los residuos de pelos tratados usados en la adsorción $\mathrm{Pb}$ (II), se encontraron algunos hallazgos:

Se observan en el espectro infrarrojo de los residuos de pelos del pelambre enzimático (figura 3 , cuadro 1) que los picos a 2962,66 (modo asimétrico de $-\mathrm{CH}_{3}$ ), 2939,52 (modo simétrico de $\left.-\mathrm{CH}_{2}\right)$, 1392,61 $\left(\mathrm{CH}_{3}\right.$ modo de flexión simétrico) y $1053,13 \mathrm{~cm}^{-1}$ (sulfonato de tensión simétrica) se desplazan a números de onda menores y cambian ligeramente las intensidades de los picos con respecto a los obtenidos en el espectro infrarrojo de los residuos de pelos tratados del pelambre enzimático (figura 4), sin embargo, el pico de la amida II $(1516,05$ $\mathrm{cm}^{-1}$ ) se desplaza a un número de onda mayor y disminuye su intensidad. Con lo que se 
demuestra que hay cambios en la estructura química del bioadsorbente bajo la acción de la hidrólisis ácida.

El proceso de pelambre produce la ruptura del enlace disulfuro de la cistina, ocasionando la formación de grupos tiol en los residuos de pelos. El grupo funcional sulfihidrilo o tiol (RSH) constituye una función química muy reactiva, esta elevada reactividad se debe a la alta nucleofilia de los iones tiolato $\left(\mathrm{RS}^{-}\right)$, los cuales existen a concentraciones significativas a valores de $\mathrm{pH}$ neutros y débilmente alcalinos. Los tioles se oxidan fácilmente generando productos con diversos estados de oxidación, por ejemplo el ácido sulfónico. La cisteína contiene un grupo tiol y puede ser oxidada por un amplio espectro de agentes oxidantes ${ }^{13}$.

Las sustancias orgánicas que poseen el grupo S-H absorben débilmente en la región de 2600 a $2550 \mathrm{~cm}^{-1}(3,85$ a 3,92 $\mu \mathrm{m})$, debido a la vibración de tensión del $\mathrm{S}-\mathrm{H}^{11}$. Esto no se observó en los tres espectros infrarrojos, debido a que el grupo tiol se ha oxidado rápidamente.

La región de bandas de 1180 a $1030 \mathrm{~cm}^{-1}$ corresponden a la cistina, en la cual se encuentran sus productos de oxidación; así, para el caso de nuestro estudio, se observaron el monóxido de cistina $\left(1072,42\right.$ y $\left.1080,75 \mathrm{~cm}^{-1}\right)$, sulfonatos de tensión simétrica $(1053,13,1049,28 \mathrm{y}$ 1041,70) y los sulfonatos de tensión asimétrica (en el rango 1200 - 1150) en los tres espectros infrarrojos (IR). Esto se genera debido a que los pelos del pelambre enzimático fueron lavados antes y después de su tratamiento, el contacto con la luz solar, el agua clorada (pelo no tratado) y el agua destilada (pelo tratado) producen la oxidación parcial de la cistina ${ }^{9}$.

En el espectro infrarrojo de los residuos de pelos tratados del pelambre enzimático antes de la adsorción de $\mathrm{Pb}$ (II) (figura 4, cuadro 1) se observan que los picos, como el monóxido de cistina $\left(1072,42 \mathrm{~cm}^{-1}\right)^{9,12,14}$, el $\mathrm{CH}_{3}$ modo de flexión simétrica $\left(1388,75 \mathrm{~cm}^{-1}\right)^{12,15,16}$ y la amida I $(1631,78)$, se desplazan a números de onda mayores mientras que los picos de la amida II $(1519,91)$, amida III $(1238,30)$ y el sulfonato de tensión simétrica $\left(1049,28 \mathrm{~cm}^{-1}\right) \mathrm{se}$ desplazan a números de onda menores con respecto al espectro IR de los residuos de pelos tratados después de la adsorción del plomo (figura 5, cuadro 1). Asimismo, en este último se observa un aumento en la intensidad de los picos del monóxido de cistina, sulfonato de tensión simétrica y del $\mathrm{CH}_{3}$ modo de flexión simétrica y una disminución de intensidad en los picos de la amida I, II y III con respecto al espectro IR de los residuos de pelos tratados antes de la adsorción $\mathrm{Pb}(\mathrm{II})$ (figura 4). Estos cambios demuestran la adsorción de plomo en el bioadsorbente.

Además, el monóxido de cistina y el sulfonato de tensión simétrica presentan en su estructura al grupo $\mathrm{S}=\mathrm{O}$. Mientras que las amidas I, II, III presentan en su estructura, estiramiento de $\mathrm{C}=\mathrm{O}$ y flexión $\mathrm{NH}$, estiramiento de $\mathrm{C}-\mathrm{N}$ y flexión plana de $\mathrm{NH}$, y estiramiento de $\mathrm{NH}$, respectivamente (cuadro 1). El espectro IR de una amida puede considerarse como una combinación de los espectros de los grupos carbonílico y amínico ${ }^{11}$. Los iones de metales pesados tales como $\mathrm{Hg}^{2+} \mathrm{y} \mathrm{Pb}^{2+}$ forman una fuerte unión con $\mathrm{CN}^{-}, \mathrm{R}-\mathrm{S}^{-},-\mathrm{SH}^{-}, \mathrm{NH}_{2}-$ e imidazol, los cuales son grupos que contienen átomos de nitrógeno y azufre (Wang y Chen) ${ }^{9}$. Por otro lado, el $\mathrm{CH}_{3}$ modo de flexión simétrica está presente en el espectro IR debido a la existencia de 
lípidos libres en la superficie de la fibra del pelo ${ }^{17}$. Existen lípidos en el cabello humano como componente libre o lípidos estructurales. Estos últimos generalmente están asociados con el CMC (complejo de membrana celular). El lípido libre del cabello humano es principalmente de origen sebáceo y existen tanto en la superficie del cabello y como en las fibras internas, el $50 \%$ de estos son ácidos grasos libres (saturados e insaturados) (Robbins ${ }^{17}$ ). Capablanca y Watt ${ }^{18}$ demostraron que los lípidos libres en la superficie de la fibra de queratina sirven para disminuir el punto isoeléctrico de la lana (pelo) y, por lo tanto, afectan el carácter de la carga de la superficie. Además, la cantidad de lípidos libres en la superficie de las fibras del cabello influirán en la fricción del cabello, la energía superficial y toda una gama de importantes propiedades que incluyen la adsorción de surfactantes y otros ingredientes en el cabello humano y fibras de lana. Asimismo, por medio del uso FTIRM en el estudio de sistemas biológicos, se establece que el CH3 modo de flexión simétrica encontrado a 1380 $\mathrm{cm}^{-1}$ representa a un lípido ${ }^{19}$. Considerando lo mencionado anteriormente y lo determinado en la comparación de los espectros IR de los residuos de pelos tratados antes y después del proceso de adsorción (figuras 4 y 5). Se puede concluir que los grupos funcionales carboxilo, amino y los grupos que contienen azufre participan en la bioadsorción del $\mathrm{Pb}$ (II). Es decir, participan estos grupos en la unión con el plomo.

Zhang ${ }^{9}$ determinó en su investigación que la adsorción de metales pesados en biomateriales de queratina se logra por los grupos funcionales hidroxilo, amino, carboxilo y grupos conteniendo azufre como sulfonatos, resultados similares a los obtenidos en esta investigación. Además, los principales grupos ionizables de unión catiónica en los biopolímeros son los carboxilos, fosfatos y sulfatos orgánicos20. En términos de la teoría ácido base, el $\mathrm{Pb}(\mathrm{II})$ es considerado un ácido blando de Lewis que puede unirse con grupos funcionales que contienen azufre, que se comportan como bases blandas de Lewis ${ }^{4}$.

\section{Análisis de difracción de rayos X (DRX)}

En el difractograma obtenido del análisis de difracción de rayos $\mathrm{X}$ de los residuos de pelos tratados antes de ser utilizados en la adsorción $\mathrm{Pb}(\mathrm{II})$, se observó que presenta una estructura o fase amorfa, es decir, no hay ordenamiento periódico, que se representa en forma de una curva y no se visualizan picos (figura 6A). Mientras que en el difractograma de los residuos de pelos tratados después ser utilizado en la adsorción $\mathrm{Pb}$ (II) se observó, además de la estructura amorfa (forma de curva), varios picos. Estos picos representan la presencia de un compuesto químico en alta concentración (figuras 7B y 7B*). La estructura amorfa solapa o encubre los picos, es decir, no permite observarlos cuando el compuesto se encuentra en poca concentración. Estos picos hallados representan al compuesto químico: $\mathrm{Pb}_{3}\left(\mathrm{CO}_{3}\right)_{2}(\mathrm{OH})_{2}$.

En el análisis DRX de los residuos de pelos tratados utilizados en el proceso de adsorción $\mathrm{Pb}$ (II), se estudiaron dos muestras, una que estuvo en contacto con la solución de plomo por 15 minutos y la otra estuvo en contacto por 30 minutos, estas son muestras tomadas del estudio cinético. Para la primera muestra se determinó que la concentración de la estructura amorfa es del 91,82 \% y del hidróxido de carbonato de plomo $\left[\mathrm{Pb}_{3}\left(\mathrm{CO}_{3}\right)_{2}(\mathrm{OH})_{2}\right]$ es de 8,18 $\%$ y para la segunda muestra se determinó que la concentración de la estructura amorfa es del $88,63 \%$ y de $\mathrm{Pb}_{3}\left(\mathrm{CO}_{3}\right)_{2}(\mathrm{OH})_{2}$ es de $11,37 \%$. Conforme el tiempo de contacto es mayor 
permite que el plomo esté más en contacto con el bioadsorbente, lo que da más probabilidades a que el plomo se una al biadsorbente, esto se da hasta un tiempo máximo en el que se satura los grupos de unión del adsorbente.

Al comparar la figura $6 \mathrm{~A}$ con las figuras $7 \mathrm{~B}$ y $7 \mathrm{~B}^{*}$ se observa que el bioadsorbente cambia su estructura después de la adsorción de $\mathrm{Pb}$ (II), que se evidencia por la formación de picos en el difractograma (picos más notorios en posición 20: $20^{\circ}-65^{\circ}$ ), esto demuestra la adsorción de $\mathrm{Pb}(\mathrm{II})$, que es corroborado con la formación de hidróxido de carbonato de plomo $\left[\mathrm{Pb}_{3}\left(\mathrm{CO}_{3}\right)_{2}\right.$ $(\mathrm{OH})_{2}$ ]. Los resultados obtenidos contrastan parcialmente con los obtenidos en el caso de la adsorción de plomo con un biopolímero compuesto de plumas y quitosano, pero son similares en la formación de hidróxido de carbonato de plomo ${ }^{21}$.

En esta investigación se dio la formación de hidróxido de carbonato de plomo debido a una microprecipitación. El cual se puede producir por una interacción entre el metal y la superficie celular a través de la formación de un complejo, seguida de su hidrolización y la precipitación del metal en forma de una especie hidrolizada en la pared celular ${ }^{22}$.

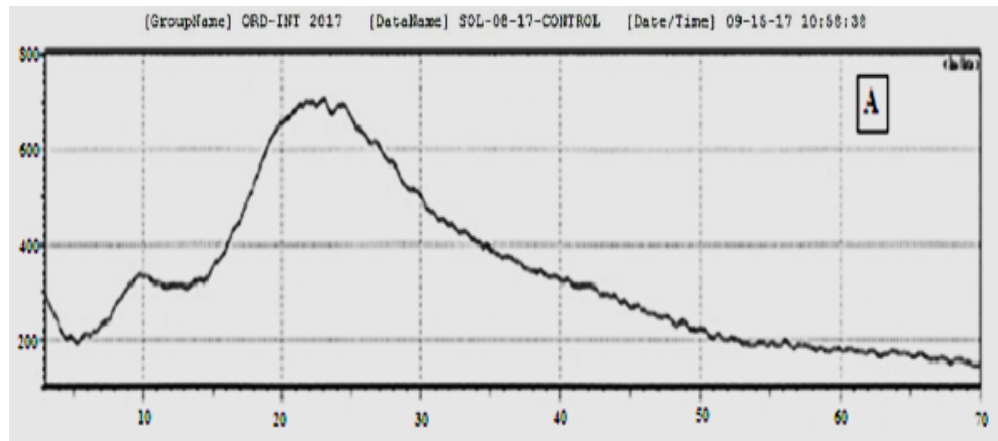

Figura 6. Difractograma obtenida del análisis de difracción de rayos $\mathrm{X}$ en residuos de pelos tratados del pelambre enzimático de la curtiembre antes de la adsorción de $\mathrm{Pb}(\mathrm{II})$.

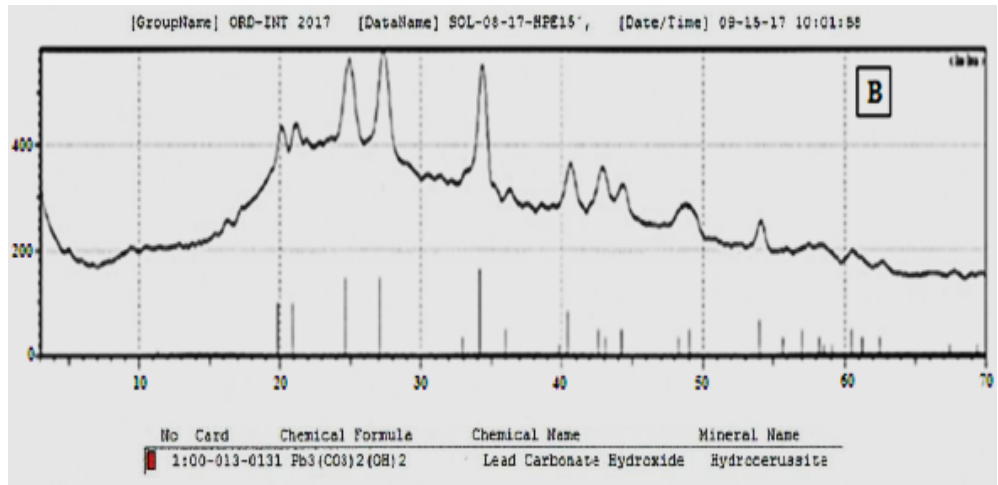

Figura 7B. Difractograma obtenida del análisis DRX de los residuos de pelos tratados del pelambre enzimático de la curtiembre después de la adsorción de $\mathrm{Pb}(\mathrm{II})$, a los 15 min de contacto. 


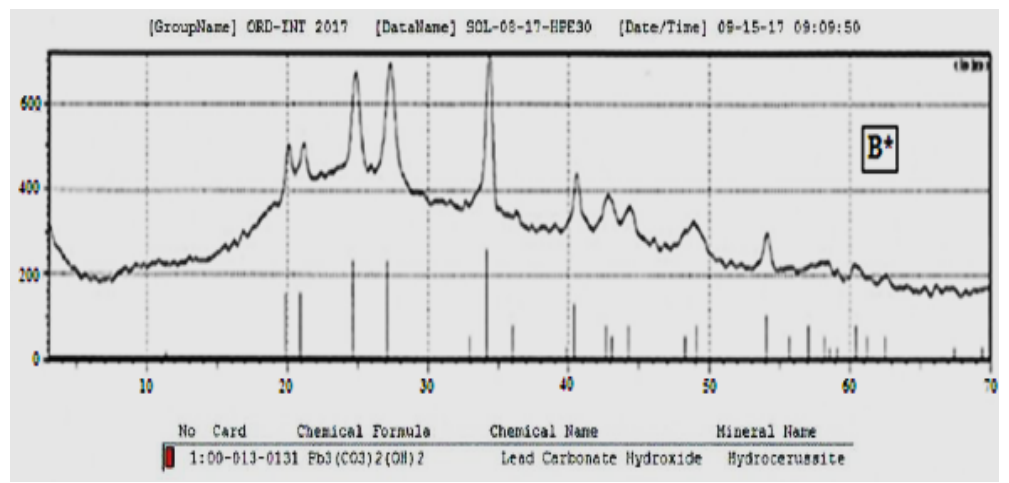

Figura 7B*. Difractograma obtenida del análisis DRX de los residuos de pelos tratados del pelambre enzimático de la curtiembre después de la adsorción de $\mathrm{Pb}(\mathrm{II})$, a los 15 min de contacto.

\section{Proceso de bioadsorción:}

En un trabajo previo ${ }^{23}$ se realizaron los estudios de las isotermas de la adsorción y de la cinética de la adsorción de $\mathrm{Pb}$ (II) sobre los residuos de pelos tratados del pelambre enzimático, a partir de los cuales se determinó que el modelo de la isoterma de la Langmuir se ajusta mejor a los resultado experimentales obtenidos $\left(\mathrm{R}^{2}=0,98\right)$, a través del cual se determinó que la capacidad máxima de adsorción de $\mathrm{Pb}$ (II) sobre los residuos de pelos tratados a pH óptimo es de 321,20 mg de $\mathrm{Pb}(\mathrm{II}) / \mathrm{g}$ de bioadsorbente. Con respecto al estudio cinético se determinó que los datos experimentales obtenidos se ajustan al modelo cinético de pseudo-segundo orden, dado que se obtiene un coeficiente de correlación promedio de $\mathrm{R}^{2}=0,98$. Además, el valor promedio de la capacidad de adsorción calculada con el modelo (qe $(\mathrm{cal})=400$ ) es similar al valor promedio de la capacidad de adsorción determinada experimentalmente (qe $(\exp )=435.24)$; lo cual corrobora la afirmación anterior. Estos resultados sugieren que el paso limitante de la velocidad es el proceso de adsorción química (quimisorción).

\section{CONCLUSIONES}

De los resultados obtenidos, se concluye que el adsorbente conseguido por medio del tratamiento de hidrólisis ácida de los residuos de pelos del proceso de pelambre enzimático de la curtiembre presenta varios grupos funcionales en la superficie que permiten la unión de $\mathrm{Pb}(\mathrm{II})$, por lo cual son materiales potencialmente útiles para usar como adsorbentes para la remoción de plomo de efluentes. Según el análisis FTIR se determinó que el bioadsorbente permite la unión del $\mathrm{Pb}$ (II) a su superficie por medio de los grupos funcionales carboxilo, amino y grupos que contienen azufre como los sulfonatos de tensión simétrica. Y por el análisis DRX se estableció la presencia y la unión del $\mathrm{Pb}$ (II) en la superficie de bioadsorbente debido a que se obtuvo un $8,18 \%$ y un $11,37 \%$ de hidróxido de carbonato de plomo. Esto se corroboró con el detector de electrones retrodispersados del SEM, que determinó la presencia de $\mathrm{Pb}$ (II) en el bioadsorbente, debido a que se generó zonas brillantes en su superficie, indicativo de la presencia de elementos con número atómico alto. Finalmente, en un trabajo 
previo, se mencionó que la capacidad máxima de adsorción fue de 321,20 mg de $\mathrm{Pb}(\mathrm{II}) / \mathrm{g}$ de bioadsorbente. Estos resultados nos permitirían aprovechar un residuo que era dispuesto en vertederos, generando contaminación, en nuevas tecnologías o tecnologías alternativas como la bioadsorción. Además, es adecuada económicamente debido a su fácil disponibilidad; así como por su relativo bajo costo de elaboración.

\section{AGRADECIMIENTO}

Se agradece al INGEMMET, Instituto Geológico, Minero y Metalúrgico, por el apoyo brindado en la realización del análisis de Difracción de Rayos X en el Laboratorio de rayos $\mathrm{X}$ del instituto. Asimismo, se agradece al Centro de Investigación en Química, Toxicología y Biotecnología Ambiental (CIQTOBIA) del Departamento Académico de Química de la UNALM por el apoyo brindado para la realización de la investigación.

\section{REFRERENCIAS BIBLIOGRÁFICAS}

1. Waihung L, Hong C, Kim L, Shu B. A comparative investigation on the biosorption of lead by filamentous fungal biomass. Chemosphere. 1999; 39: 2723-2736.

2. Wong $\mathrm{K}$, Lee $\mathrm{C}$, Low $\mathrm{K}$, Haron $\mathrm{M}$. Removal of $\mathrm{Cu}(\mathrm{II})$ and $\mathrm{Pb}$ (II) by tartaric acid modified rice husk from aqueous solutions. Chemosphere. 2003; 50: 23-28.

3. Lavado MC, Sun KM, Bendezú S. Adsorción de plomo de efluentes industriales usando carbones activados con H3PO4. Rev Soc Quím Perú. 2010; 76 (2): 165-178.

4. Valencia RJS, Castellar OGC. Predicción de las curvas de ruptura para la remoción de plomo (II) en disolución acuosa sobre carbón activado en una columna empacada. Rev Fac Ing Univ Antioquia. 2013; 66: 141-158

5. Goel J, Kadirvelu K, Rajagopal Ch, Garg VK. Removal of lead (II) by adsorption using treated granular activated carbon: Batch and column studies. J Hazard Mater. 2005; B125: 211-22

6. Hawkins TR. Remoción de cromo total de efluentes de la industria de galvanoplastia por adsorción con gel SOW-Fe. [Tesis para optar el título de Ingeniero Ambiental]. Lima: Universidad Nacional Agraria La Molina, Perú; 2012.

7. García Roig M, Ramírez PFI, Manzano MT. Bioadsorción de metales pesados de aguas ácidas de minas (y II) sobre residuos con queratina (pelos de cerdo, pluma de ave) y quitina (caparazones de crustáceos). Ingeniería Química. 2004; 402: 184 - 204.

8. Ghosh A, Collie SR. Keratinous Materials as Novel Absorbent Systems for Toxic Pollutants. Def Sci J. 2014; 64(3): 209-221.

9. Zhang $\mathrm{H}$. Biosorption of heavy metals from aqueous solutions using keratin biomaterials. [Tesis doctoral]. Barcelona: Universidad Autónoma de Barcelona; 2014.

10. Larenas UC, Andrango D, Inga P. Estudio isotérmico de biosorción de plomo en aguas utilizando residuos vegetales. La Granja. 2008; 8(2): 3-8.

11. Conley RT. 1979. Infrared Spectroscopy. 2nd. Ed. Boston, Massachusetts: Allyn and Bacon Inc. 1979. 
12. Bantignies JL, Carr GL, Lutz D, Marull S, Williams GP, Fuchs G. Chemical imaging of hair by infrared microspectroscopy using synchrotron radiation. J Cosmet Sci. 2000; 51: 73-90

13. Sardi PF. Caracterización de la acidez y nucleofilia de tioles de bajo peso molecular y tioles proteicos. [Tesis de grado para obtener la licenciatura en Bioquímica]. Montevideo: Universidad de la República; 2011.

14. Dankers LM. Physical analysis of human hair. [Masters Theses]. Rolla, Missouri: Missouri University of Science and Technology; 2007.

15. Taddei P, Monti P, Freddi G, Arai T, Tsukada M. Binding of $\mathrm{Co}(\mathrm{II})$ and $\mathrm{Cu}(\mathrm{II})$ cations to chemically modified wool fibres: an IR investigation. J Mol Struct. 2003; 650: 105-113.

16. Lau K, Hedegaard M, Kloepper J, Paus R, Wood B, Deckert V. Visualization and characterization of defined hair follicle compartments by Fourier transform infrared (FTIR) imaging without labeling. J Dermatol Sci. 2011; 63: 191-198.

17. Panayiotou H. Vibrational spectroscopy of keratin fibres a forensic approach. [Tesis de Ph. D.]. Brisbane: Queensland University of Technology; 2004.

18. Robbins CR. Chemical composition of different hair types. En Chemical and physical behavior of human hair. New York: Springer-Verlang; 2002. p105-176.

DOI 10.1007/978-3-642-25611-0_2.

19. Barraza GG, De la Rosa LA, Martínez MA, Castillo M H, Cotte M, Alvarez PE. La microespectroscopía de infrarroja con transformada de Fourier (FTIRM) en el estudio de sistemas biológicos. Rev Latinoamer Quím. 2013; 41(3):125-148.

20. García RM, Ramírez PFI, Manzano MT. Bioadsorción de metales pesados de aguas ácidas de minas (I): Sobre residuos de levaduras cerveceras. Ingeniería Química. 2003; 400: 158-168.

21. Ratna Kumaria A, Sobhab K. Removal of lead by adsorption with the renewable biopolymer composite of feather (Dromaius novaehollandiae) and chitosan (Agaricus bisporus). Environmental Technology \& Innovation. 2016; 6: 11-26.

22. Viglio F, Beolchini F. Removal of metals by biosorption: a review. Hydrometallurgy.1997; 44:301-316.

23. Palomino Amorín C, Vargas PR, Visitación FL. Aprovechamiento de pelos de vacuno del proceso de pelambre enzimático de las curtiembres en la remoción de plomo. Rev Soc Quím Perú. 2016; 82 (2). 